\title{
Behavior of Weak Polyelectrolyte Brushes in Mixed Salt Solutions
}

\author{
Joshua D. Willott, ${ }^{*},, \# \oplus$ Timothy J. Murdoch, ${ }^{+, \# \odot ~ F r a n s ~ A . ~ M . ~ L e e r m a k e r s, ~}{ }^{\S}$ and Wiebe M. de $\operatorname{Vos}^{\dagger}$ \\ ${ }^{\dagger}$ Membrane Science and Technology, Mesa+ Institute for Nanotechnology, University of Twente, Enschede 7500 AE, The \\ Netherlands \\ ${ }^{*}$ Priority Research Centre for Advanced Particle Processing and Transport, University of Newcastle, Callaghan, NSW 2308, Australia \\ ${ }^{\S}$ Physical Chemistry and Soft Matter, Wageningen University and Research, Wageningen 6708 WE, The Netherlands
}

\section{Supporting Information}

ABSTRACT: Hydrophilic and hydrophobic weak polybasic brushes immersed in aqueous solutions of mixed salt counterions are considered using a mean-field numerical selfconsistent field approach. On top of the solvent quality of the polymer, the counterion-solvent interactions are accounted for by implementing Flory-Huggins interaction parameters. We show that ion specificity within the brush can bring about large changes in conformation. It is found that the collapse transition of hydrophobic, weak polyelectrolyte brushes

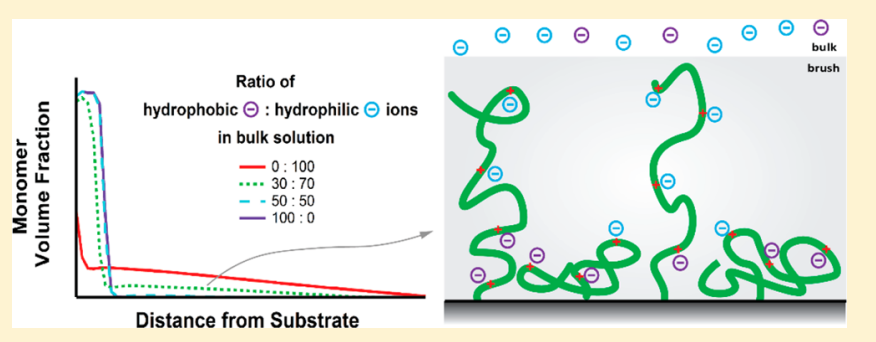
features an intermediate two-phase state wherein a subset of chains are collapsed in a dense layer at the substrate, while the remainder of chains are well-solvated and strongly stretched away from the it. Besides $\mathrm{pH}$ and ionic strength, solvent quality of counterions and the composition of ions in the solvent are important control parameters for the behavior of polyelectrolyte brushes. Increasingly hydrophobic counterions penetrate deeper within the brush and stabilize the collapsed region, while hydrophilic counterions do the opposite.

\section{INTRODUCTION}

Weak polyelectrolyte brushes are responsive to environmental conditions including $\mathrm{pH}$, ionic strength, and type of ionic species in solution. ${ }^{1-5}$ The ability to control brush conformation by an environmental stimulus makes these responsive brushes ideal for many applications including microfluidics, reversible colloidal stabilization, biosensing, and chemical gating. ${ }^{6}$ The swelling response of hydrophilic and hydrophobic weak polyelectrolyte brushes in monovalent salt solutions has been well-characterized, and close agreement between theory and experiment is possible. ${ }^{7,8}$ However, many theories are incomplete. For example, until recently, ${ }^{9,10}$ known specific ion effects in weak polyelectrolyte brushes were not accounted for by theory.

Analytical $^{11-13}$ and numerical ${ }^{10,14-18}$ self-consistent field (SCF) theories along with molecular theories ${ }^{19-22}$ have been applied to weak polyelectrolyte brushes. Close agreement between neutron reflectivity fits of poly(acrylic acid) (PAA) brushes and predictions from analytical SCF theory was reported by Sudre et al. ${ }^{23}$ Mahalik and co-workers were able to qualitatively reproduce the volume fraction profile of a poly(2-dimethylamino)-ethyl methacrylate brush using nSCF theory. ${ }^{24}$ The molecular theory of Léonforte et al. ${ }^{22}$ has reproduced the equilibrium $\mathrm{pH}$ and ionic strength response of an acidic PAA and a basic poly(2-vinylpyridine) brush, while also providing insight into the kinetics of brush swelling and collapse that supports experimental data. Recently, we have shown that trends in the $\mathrm{pH}$, ionic strength, and specific ion response of a weakly basic poly(2-diisopropylamino)ethyl methacrylate brush as measured by in-situ ellipsometry ${ }^{4,5}$ and neutron reflectometry ${ }^{10}$ can be qualitatively matched using a nSCF theory that assigns a Flory-Huggins interaction parameter (with the solvent) to both the monomer segments and salt counterions. ${ }^{10}$

All these experimental and theoretical studies have led to a great deal of understanding on the responsive properties of weak polyelectrolyte brushes. The effect of $\mathrm{pH}$ in these systems is relatively straightforward. For polybasic brushes in acidic solution, the polymer protonates (with the degree dependent on the $\mathrm{p} K_{\mathrm{a}}$ of the polymer and the ionic strength), and the increase in charge results in chain extension (due to electrostatic repulsion) and brush swelling (due to solvent and counterion uptake). The opposite is true in alkaline solutions, where the brush deswells and the polymer chains contract. The behavior of weak polyelectrolyte brushes to variations in salt concentration is more complicated. ${ }^{8,22}$ To compensate for the energetically unfavorable electrostatic repulsions between chains, the brush responds via three mechanisms: the acid-base equilibria can shift to the uncharged state, chains can extend with the cost of losing conformational entropy, or counterions can be confined within the brush at the expense of translational entropy. At low salt concentration, the first mechanism dominates and the brush is uncharged, and so less extended chain conformations are

Received: October 20, 2017

Revised: December 22, 2017

Published: January 17, 2018 
favored. With increasing ionic strength, counterion confinement is more favorable, allowing the brush to charge while simultaneously swelling due to the increased osmotic pressure (osmotic brush). At higher salt concentrations, the brush is fully ionized, and charge screening effects result in brush collapse (salted brush). With all other brush characteristics being equal, hydrophilic polymers swell to a greater extent and also deswell less because polymer-solvent interactions are more favored for hydrophilic polymers compared to hydrophobic ones, which undergo collapse in poor solvent conditions.

Weak polybasic polymers also experience clear specific anion (Hofmeister) behavior at salt concentrations greater than 10-50 mM. ${ }^{5,10,25,26}$ For these brushes, in the presence of strongly hydrated (kosmotropic) anions, brush swelling is large, while the degree of swelling is much lower in the presence of weakly hydrated (chaotropic) anions. In our previous work, we show that the specific anion response of a hydrophobic weak polybasic brush can be accounted for by considering the degree of hydration of the counterion. ${ }^{10}$

Experimentally, it is known that the swelling and structural behavior of weak polyelectrolyte brushes depend on numerous factors: $\mathrm{pH}$, ionic strength, type of salt ions, valence of salt ions, hydrophobicity of monomers, temperature, polymer grafting density, and electric fields. Nowadays, all of these factors can be accurately and efficiently evaluated for polyelectrolyte brush systems. However, one important question that remains unexplored is how will polyelectrolyte brushes respond in mixed salt solutions (or real-world environments). Here we report on how weak polyelectrolyte brushes respond in mixed salt solutions and present scenarios wherein one type of salt controls the brush behavior in mixed salt solutions. We adapt our existing nSCF theory ${ }^{10}$ for weak polybasic brushes to study salt solutions composed of different types of salt ions. Specifically, we model brush behavior for varying percentages of monovalent chaotropic (weakly hydrated, hydrophobic) counterions and monovalent kosmotropic (strongly hydrated, hydrophilic) counterions present in the bulk solution. In this work we show that our nSCF theory can be used as a predictive tool in the planning and developing of well-targeted experimental studies. Moreover, we show how solvents containing mixtures of salt provide additional pathways to tune the responsive behavior of polybasic brushes.

\section{THEORETICAL MODELING APPROACH}

Over the years, numerical self-consistent field (nSCF) theories have simulated the conformational and structural features of ionizable (weak) polyelectrolyte brushes, and many of these predictions have been verified experimentally. ${ }^{10,23,24}$ The predictions of nSCF theory align excellently with those made by molecular dynamics simulations and are more computationally efficient by several orders of magnitude. ${ }^{27}$ The lattice model employed here is that of Scheutjens and Fleer, ${ }^{28}$ which is described in detail elsewhere, ${ }^{28-30}$ so only essential theory and assumptions will be discussed below.

One point that we feel is important to emphasize here are the limitations of our model. nSCF theories suffer from a lack of chemical detail because the shape and size of all species are defined by the lattice. As such, complex models, like the one implemented here, are not intended to quantitatively predict or replicate experimental results but instead elucidate trends ${ }^{31}$ and to provide qualitative insight into brush behavior.
Accurate simulation of polymer brushes requires the Edwards diffusion equation for polymer chains immersed in inhomogeneous systems to be solved: ${ }^{32}$

$$
\frac{\delta G(\mathbf{r}, s \mid 1,1)}{\delta s}=\left(\frac{1}{6} \nabla^{2}-u(\mathbf{r})\right) G(\mathbf{r}, s \mid 1,1)
$$

where the Green's function $G$ is the statistical weight of all possible chain conformations with segment $s^{\prime}=1$, next to the substrate surface $\left(\mathbf{r}_{z}=1\right)$ and segment $s^{\prime}=s$, at coordinate $\mathbf{r}$, and $u(\mathbf{r})$ is the dimensionless segment potential. $G$ is closely related to the chain partition function (when $s=N$, the total number of segments) and hence the Gibbs free energy of the system. The segment potential mimics the excluded volume interactions, while also accounting for the solvent quality and the electrostatic interactions (which are discussed a little later). Equation 1 has generally no exact analytical solution but, as shown by Scheutjens and Fleer, ${ }^{28}$ can be implemented in a rather general formalism that can be solved numerically with high accuracy. This method makes use of lattice approximations and implies a noticeable change of the chain model; instead of the Gaussian chain model, the lattice-freely jointed chain model is used for eq 1 . The difference between these chain models is only important when finite chain extensibility is considered. Gaussian chains can stretch beyond the contour length, while freely jointed chains on a lattice cannot.

The self-consistent field model of Scheutjens and Fleer hence focuses on the evaluation of the statistical weight of all possible and allowed freely jointed chain conformations of the endtethered polymer chains, where a collection of these chains forms a laterally homogeneous brush. In the current model, the brush is completely immersed in a molecular solvent (with states $\mathrm{H}_{2} \mathrm{O}, \mathrm{OH}^{-}$, and $\mathrm{H}_{3} \mathrm{O}^{+}$) that contains cationic and anionic salt ions. Within the freely jointed chain model there exists an efficient propagator formalism that leads directly to the single chain partition function (needed for the free energy and for normalization of the density profiles) and produces routinely the segment density profiles for the polymer brush. The current nSCF work makes no prior assumptions concerning the shape of the segment potential profile and thus allows for deviations from analytical forms, such as the presence of (micro)phase separation, ${ }^{33}$ to be captured. Three distinct components are known to influence the segment potentials. First, there is a socalled Lagrange contribution of which its value is coupled to the (in) compressibility condition $\sum_{i} \varphi_{i}=1$, where the index $i$ runs over all "segment" $(\varphi)$ types in the system (including solvent and ions). The second contribution is due to the short-ranged interaction (solvency effects), and the final contribution is due to the electrostatic contributions (similarly as in PoissonBoltzmann theory). Typically, the segment potential is made dimensionless by the thermal energy $k T$. Here and below we follow this habit for all energy units.

The short-ranged molecular interactions are parametrized by Flory-Huggins nearest-neighbor (dimensionless) interaction parameters $\chi_{i j}$, while the number of contacts between components $i$ and $j$ is estimated using the Bragg-Williams mean-field approximation. The contribution due to charges involves a term proportional to the segment valence, the elementary charge $e$, and the electrostatic potential, $\psi(z)$. In the case of monovalent ions $z_{i}= \pm 1$, the segment potential is given by (plus or minus) the dimensionless segment potential $\Psi(z)=$ $e \Psi(z) / k T$. Evaluating this electrostatic potential requires solving of the Poisson equation: ${ }^{34}$ 


$$
\nabla^{2} \Psi(z)=-\frac{1}{\varepsilon_{0}} q(z)
$$

Here, $q(z)$ is the number distribution of charges, where cations add positively and anions negatively to this quantity, and $\varepsilon_{0}$ is the dielectric constant of the solution. It is assumed that the dielectric permittivity is equal to that of water throughout the system.

The solvation of a polymer brush, and hence its swelling, depends strongly on the value and the sign of the overall virial coefficient and reads $v=v_{\text {bare }}+v_{\mathrm{el}}$. The bare virial coefficient $v_{\text {bare }}$ is linked to the solvent quality $\chi$, (via, $v_{\text {bare }}=1-2 \chi$ ), and the electrostatic contribution $v_{\mathrm{el}}$ is inversely proportional to the concentration of mobile salt ions $\varphi_{\mathrm{s}}$ and a quadratic function of the charge density $\alpha$ in the brush: $v_{\mathrm{el}}=\alpha^{2} / \varphi_{\mathrm{s}}$. For polyelectrolytes in good solvents, the bare virial coefficient is often negligible compared to the electrostatic contribution. For hydrophobic polyelectrolytes, the overall virial coefficient does not necessarily dominate the electrostatic contribution, and the switching of the sign of the overall virial coefficient is expected to have important consequences for the brush structure.

For weak polyelectrolyte brushes, the degree of dissociation (and thus the value of $\alpha$ ) is dependent on $\mathrm{pH}$, ionic strength, and the local electrostatic potential, ${ }^{15}$ which is modeled using a two-state theory. ${ }^{32}$ For a weakly basic polycation, the monomer $\mathrm{B}$ can exist in neutral unprotonated state and a cationic protonated state: $\mathrm{B}+\mathrm{H}_{3} \mathrm{O}^{+} \rightleftharpoons \mathrm{BH}^{+}+\mathrm{H}_{2} \mathrm{O}$. In this model we assume a monomeric $\mathrm{p} K_{\mathrm{a}}$ of 7 for symmetry and to limit the influence of $\mathrm{pH}$ on the ionic strength. The autodissociation of water is implemented as $2 \mathrm{H}_{2} \mathrm{O} \rightleftharpoons \mathrm{OH}^{-}+\mathrm{H}_{3} \mathrm{O}^{+}$with a $\mathrm{pK}$ w 14. The degree of protonation, $\alpha$, at location $z$ then follows from $\alpha(z)=\frac{K_{\mathrm{a}}}{K_{\mathrm{a}}+\left[\mathrm{H}^{+}\right] \mathrm{e}^{-\Psi(z)}}$, where $\Psi(z)$ represents the local dimensionless electrostatic potential. ${ }^{15}$ Consequently, the degree of dissociation can vary perpendicularly to the substrate surface, which is important for low ionic strengths where the Debye screening length is large.

The optimal brush structure is found after optimization of the mean-field energy. This optimization process is effectively implemented by a formalism that leads to the so-called selfconsistent field results: (i) the maximization of the free energy to the Lagrange field gives the incompressibility rule (see above), (ii) the maximization of the free energy with respect to the segment potentials leads to the rule that segment densities should be computed from specified segment potentials (as explained above, the freely jointed chain propagator formalism effectively does this), and (iii) the optimization of the free energy to the segment densities gives the protocol to compute the volume fractions from the segment densities. We note that when the electrostatic potentials follow from the Poisson equation we know that we have also optimized the free energy with respect to charge distribution. Any solution that obeys all the rules are said to have potentials and densities that are consistent to each other and, hence, are referred to as the selfconsistent solution. This point is routinely found numerically by an iterative procedure which is only stopped when seven significant digits are obtained for both the potentials and densities of all molecular species. The CPU time is typically short (seconds to minutes rather than hours), and the computer program (SFbox) runs on a desktop PC.

\section{MODEL IMPLEMENTATION}

Our model of a weak polybasic brush has been implemented using a one gradient planar lattice with the key parameters summarized in Figure 1. Each lattice site has a size of $0.5 \mathrm{~nm}$

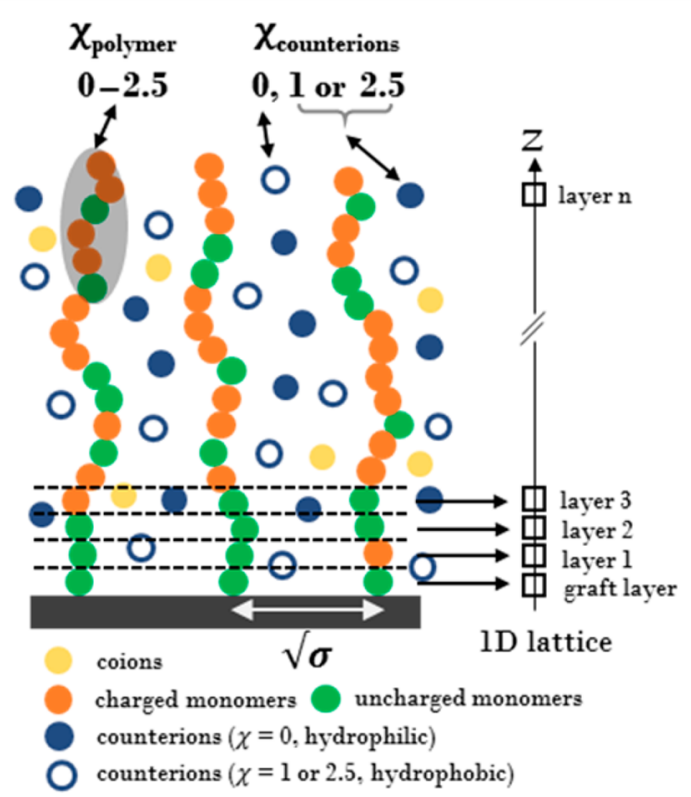

Figure 1. Schematic illustration of the model and coordinate system employed for nSCF calculations. The only relevant coordinate is perpendicular to the substrate (the $z$ direction). Each lattice layer parallel to the substrate is defined by a volume fraction of each species (polymer, co-ions, counterions, and water) with the size of each species equal to that of the size of a lattice site $(0.5 \mathrm{~nm})$. The grafting density is fixed by the volume fraction of polymer grafting points in the surface layer. Monomers and water can be either neutral or charged. The hydrophobicity of the polymer and the counterions is set by their respective Flory-Huggins $(\chi)$ interaction parameters with the solvent $(\chi<0.5=$ hydrophilic, $\chi>0.5=$ hydrophobic $)$.

(volume of $0.125 \mathrm{~nm}^{3}$ ). The polymer chain length, $N$, in the brush is set to 100 , and the grafting density, $\sigma$, is 0.025 chains per lattice site $\left(0.1 \mathrm{~nm}^{-2}\right)$, well within the brush regime where the height of the brush significantly exceeds the distance between grafting points. Polymer solvent quality (interaction between polymer and solvent) was varied from 0 (good solvent) to 2.5 (very poor solvent) in 0.5 unit increments; $\chi_{\text {polymer }}=0.5$ corresponds to the Flory-Huggins theta condition. This range is realistic; poly(2-dimethylamino)ethyl methacrylate, a relatively hydrophilic weak polybase, has a $\chi_{\text {polymer }} \sim 0.6$ at $25{ }^{\circ} \mathrm{C}$, with values $>2$ achieved when it is copolymerized with hydrophobic butyl methacrylate. ${ }^{35}$ The bulk ionic strength and $\mathrm{pH}$ in the system are controlled by fixing a volume fraction of positively charged co-ions, $\phi_{\text {co-ions, }}$ and $\mathrm{H}_{3} \mathrm{O}^{+}$, respectively, while the volume fraction of counterions, $\phi_{\text {counterions, }}$ is set by the electroneutrality constraint for the reference bulk solution. Converting from ion volume fraction to molarity is easy; simply multiply by the molarity of bulk water (55 M). Importantly, this is not an exact conversion due to necessary compromises in setting the model's lattice parameters.

Specific ion and mixed salt effects are approximated by assigning Flory-Huggins interaction parameters to the

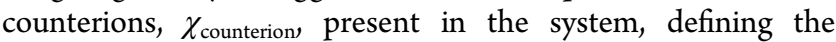
counterion-solvent interaction. Values of $\chi_{\text {counterion }}<0.5$ reflect 


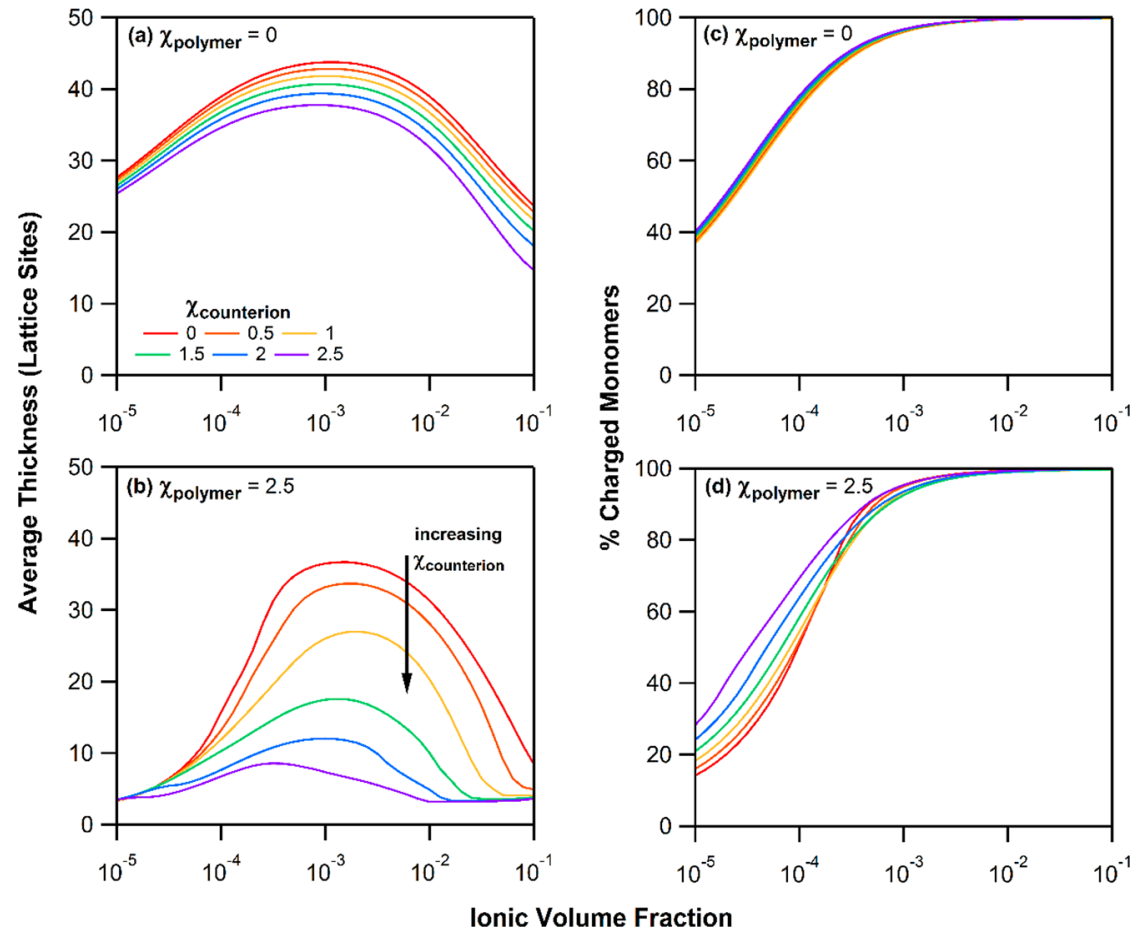

Figure 2. Average brush thickness as a function of ionic strength for a hydrophilic $\left(\mathrm{a}, \chi_{\text {polymer }}=0\right)$ and hydrophobic $\left(\mathrm{b}, \chi_{\text {polymer }}=2.5\right)$ weak polybasic brush and the corresponding percentage of charged monomers $(c, d)$. Data calculated at constant $\mathrm{pH}$ value of 4 ( 3 units below $\mathrm{p} K_{\mathrm{a}}$ of monomer units) for increasing values of $\chi_{\text {counterion }}$ corresponding to increasing counterion hydrophobicity.

strongly hydrated kosmotropes, while $\chi_{\text {counterion }}>0.5$ (up to 2.5) are for increasingly chaotropic or weakly hydrated counterions. It is important to note that we make no assumption into the origin of the specific ion interactions, e.g., dispersion forces or Collins law of matching water affinities. ${ }^{36}$ Our recent publication showed that the addition of the $\chi_{\text {counterion }}$ parameter was sufficient to qualitatively reproduce the experimentally observed specific ion response of a weak poly(2-diisopropylamino)ethyl methacrylate brush. ${ }^{10}$ In the presence of strongly hydrated acetate anions, the brush behavior was qualitatively reproduced with $\chi_{\text {counterion }}=0$, while values of $\chi_{\text {counterion }}$ of 1 and 2.5 matched the trends seen for the increasingly chaotropic (or weakly hydrated) nitrate and thiocyanate ions. ${ }^{10}$ In the present work, we study the influence of mixed salt solutions (mixtures of two different types of salt) on weak polyelectrolyte brush behavior by varying the ratio of two distinct counterion species with different Flory-Huggins parameters present in solution. In all simulations, the solution $\mathrm{pH}$ and co-ion concentration have been kept constant. We define the average brush thickness as the first moment of the polymer volume fraction profile. Counterion confinement within the brush is determined by calculating the excess amount of the given ion, $\theta_{i}^{\text {brush }}$, in the system, $\theta_{i}=\sum_{i} \varphi_{i}(z)-$ $\varphi_{i}^{\text {bulk }}$, as shown in Figure S1.

\section{RESULTS AND DISCUSSION}

This section covers three main topics. First, in order to set the foundations, brush response to the variation of a single type of salt counterion is discussed, revealing that brush conformation is dependent on both polymer and counterion hydrophilicity. Second, a wide range of mixed salt solutions are considered, and the overall confinement of hydrophilic versus hydrophobic counterions within the brush is explored together with the consequences this has on average brush thickness and conformation. Third, the precise location of hydrophilic and hydrophobic counterions confined within the brush is investigated.

Influence of Single Salt Solutions. As mentioned above, to begin we will study the response to varying ionic strength of a weak polybasic brush immersed in a solvent consisting of only a single type of salt counterion. We study ionic volume fractions ranging from $1 \times 10^{-5}$ to $1 \times 10^{-1}(\sim 0.55$ to 5500 $\mathrm{mM}$ ). In Figure 2, we present the average brush thickness and average charge (\% charged monomers) for both a hydrophilic $\left(\chi_{\text {polymer }}=0\right)$ and a hydrophobic $\left(\chi_{\text {polymer }}=2.5\right)$ polymer for $\mathrm{pH}$ $=4$. Choosing $\mathrm{pH} 4$ is significant as $\mathrm{pH} \ll \mathrm{p} K_{\mathrm{a}}$, so we can anticipate that in the absence of any electrostatic potential the degree of charging of the brush would be extremely close to unity at high enough bulk ionic strengths. At higher $\mathrm{pH}$ values, closer to and above the $\mathrm{p} K_{a}$, the percentage of charged monomer decreases. ${ }^{10}$ For both the hydrophilic and hydrophobic polymers, the variation in overall brush thickness is nonmonotonic, as expected from existing theories ${ }^{11,19}$ and experiments $^{3,5}$ for weak polyelectrolyte brushes.

At low ionic strength $\left(<10^{-3}\right)$, the percentage of charged monomers within the brush is low, but with more added salt, the brush charge increases, reaching its maximum value at an ionic volume fraction of $\sim 10^{-3}$ (see Figure $2 \mathrm{c}, \mathrm{d}$ ). The brush thickness initially follows the same trend: increasing with salt concentration until a maximum value, as a result of the greater electrostatic repulsion between chains and the uptake of counterions and associated solvent molecules by the brush from the bulk solution. This is the osmotic brush regime. ${ }^{11}$ However, at higher ionic strengths, $>10^{-3}$, the average brush thickness begins to decrease as electrostatic screening effects become dominant (smaller Debye screening length), the salted brush regime, ${ }^{11}$ and in poor solvents (hydrophobic polymers) collapsed polymer conformations are favored. For experimental 
studies on strongly charged polybasic brushes, ion-specific reswelling of the brush has been reported at very high ionic strengths and is attributed to charge reversal of the polymer chains; ${ }^{37,38}$ this overcharging effect is not observed in our work.

The influence of polymer hydrophobicity on brush thickness is strikingly clear. In Figure $2 \mathrm{~b}$ for the hydrophobic polymer, ionic strength and counterion hydrophilicity have a much more pronounced effect on brush thickness. Even when uncharged the hydrophilic polymer is swollen, as expected for good solvent conditions. With increasing charge, brush thickness increases, but finite chain extensibility and excluded volume interactions result in limited chain extension, ${ }^{39}$ so the additional swelling is minimal. For hydrophobic polymers, however, when uncharged at low salt or when the charge is screened at high salt, favored polymer-polymer interactions result in collapsed brush conformations. The brush thickness increases and reaches a maximum at intermediate salt concentrations, but the inherent hydrophobicity of the polymer means that the degree of swelling is substantially less than for the more hydrophilic polymer. In Figure 2, for all cases, average brush thickness decreases with increasing values of $\chi_{\text {counterion }}$ (counterion hydrophobicity), with the hydrophobic polymer being most sensitive to changes in $\chi_{\text {counterion }}$. For both polymers, the average charge is essentially invariant with $\chi_{\text {counterion }}$ above ionic volume fractions of $\sim 10^{-3}$ (see Figure $2 \mathrm{c}, \mathrm{d}$ ), suggesting that counterion hydration plays a significant role in the observed brush thickness response in salted brush regime. Indeed, comparing the results presented in Figure 2 with our previous experimental work on three weak polybasic brushes of varying hydrophobicity reveals that the trends for both the polymer hydrophobicity, ${ }^{4}$ and the specific anion response, ${ }^{5}$ match up closely.

In Figure 3, we present predicted monomer volume fraction profiles for hydrophilic and hydrophobic brushes in the presence of counterions of increasing hydrophobicity $\left(\chi_{\text {counterion }}\right.$ $=0,1$, and 2.5) over a range of solution ionic strengths. As expected from Figure 2a, hydrophilic polymers are always highly extended for all conditions. However, the interesting behavior occurs for the hydrophobic polymers. Specifically, as the hydrophobic polyelectrolyte brush collapses, either by increasing ionic strength or by increasing counterion hydrophobicity, an intermediate two-phase state appears in the polymer density profile. Here, a subset of chains are collapsed in a dense layer near the substrate, and the remainder of chains are strongly extended-microphase segregation of polymer chains within the brush. Theories of, ${ }^{4-43}$ and experiments on, ${ }^{44,45}$ both strongly and weakly charged polyelectrolyte brushes show that in poor solvents nanoscale lateral phase separation can occur, resulting in the formation of bundledcylinder, micelle, and maze-like structures. Two- or threegradient $(2 \mathrm{D}$ or $3 \mathrm{D})$ lattice $\mathrm{nSCF}$ calculations can be applied to study the lateral (in)homogeneity of thin brush layers. Studying this behavior requires careful consideration of the interactions between the polymer and the substrate surface. For example, when the affinity of the polymer for the substrate is sufficiently attractive, lateral stability is expected, i.e., complete wetting of the condensed polymer phase. Moreover, the thickness of the collapsed polymer phase will be important. This is indeed a complex problem and would require a separate dedicated work studying the microphase segregation. In this article we focus on salt mixtures and how the different ions interact with polyelectrolyte brushes.
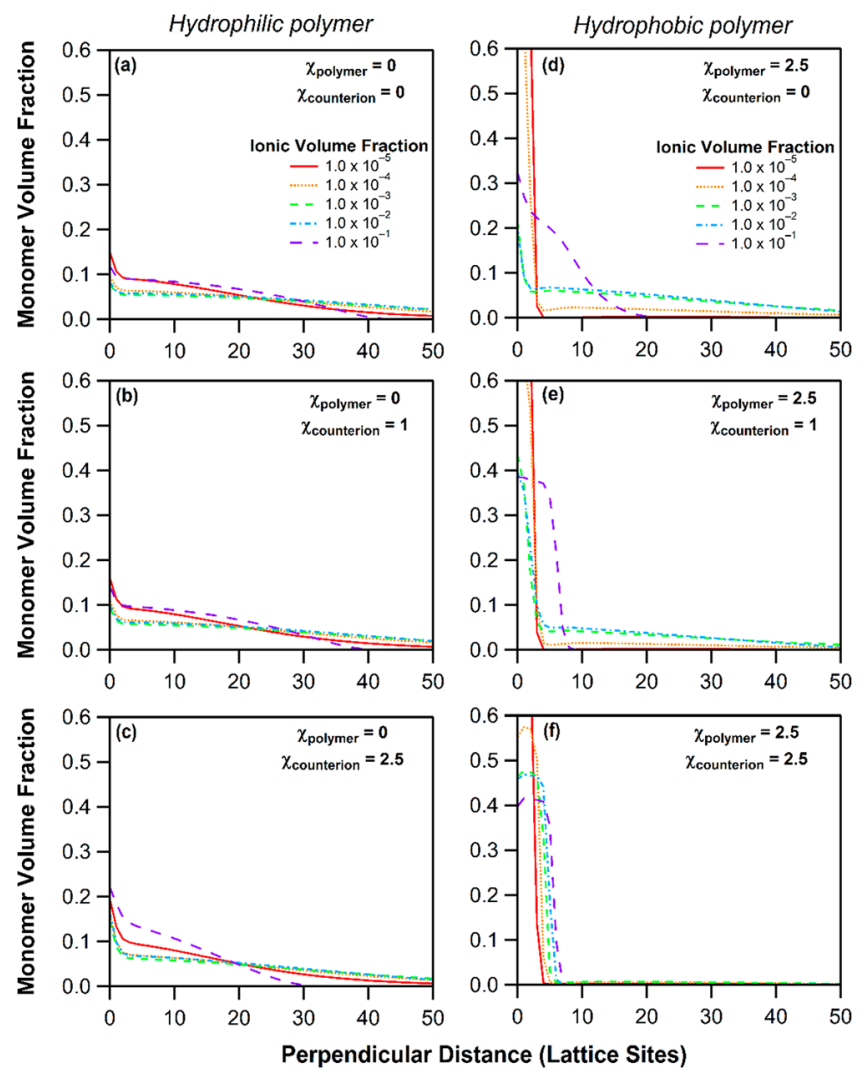

Figure 3. Monomer volume fraction profiles for a hydrophilic (a, b, c, $\left.\chi_{\text {polymer }}=0\right)$ and hydrophobic $\left(\mathrm{d}, \mathrm{e}, \mathrm{f}, \chi_{\text {polymer }}=2.5\right)$ as a function of $\chi_{\text {counterion }}(0,1$, and 2.5$)$ and ionic volume fraction.

In our recent publication we report on the good agreement between $\mathrm{nSCF}$ predictions and neutron reflectometry results for a hydrophobic weakly basic poly(2-diisopropylamino)ethyl methacrylate brush. ${ }^{10}$ This together with our previously published ellipsometry and QCM-D findings ${ }^{4,5}$ provides strong validation of the assumptions within our nSCF model. Specifically, the choice to impose a solvent quality $\chi$ parameter on the anion $\left(\chi_{\text {counterion }}\right)$ to simulate its degree of hydration is justified. In the next section, we extend our nSCF model to investigate mixed salt systems by studying the effect of varying the ratios of hydrophilic and hydrophobic counterions present in solution, showing that under certain conditions brush behavior can be dominated by the presence of small volume fractions of hydrophobic counterions.

Influence of Mixed Salt Solutions. The brush salt response when immersed in mixtures of varying compositions of hydrophilic $\left(\chi_{\text {counterion }}=0\right)$ and hydrophobic $\left(\chi_{\text {counterion }}=2.5\right)$ counterions for $\mathrm{pH}=4$ is presented in Figure 4 . We have also investigated brush response to mixtures of the counterions of $\chi_{\text {counterion }}=0$ and 1 ; the influence on average brush thickness is smaller and presented in Figure S2. Calculations were also performed for higher $\mathrm{pH}$ values of 5.5 and 7 (where the monomer $\mathrm{pK}_{\mathrm{a}}$ value is 7). Here, as the solution $\mathrm{pH}$ increases, the percentage of charged monomers decreases, for the same bulk ionic strength. Importantly, the overall trends in counterion confinement and brush response when immersed in mixed salt solutions that will be discussed in this section are the same. Considering the single salt nSCF calculations, it is not surprising that the influence of mixed salts on the average thickness of the hydrophilic weak polybasic brush is small (see Figure 4a). Conversely, dramatic effects are seen for the 


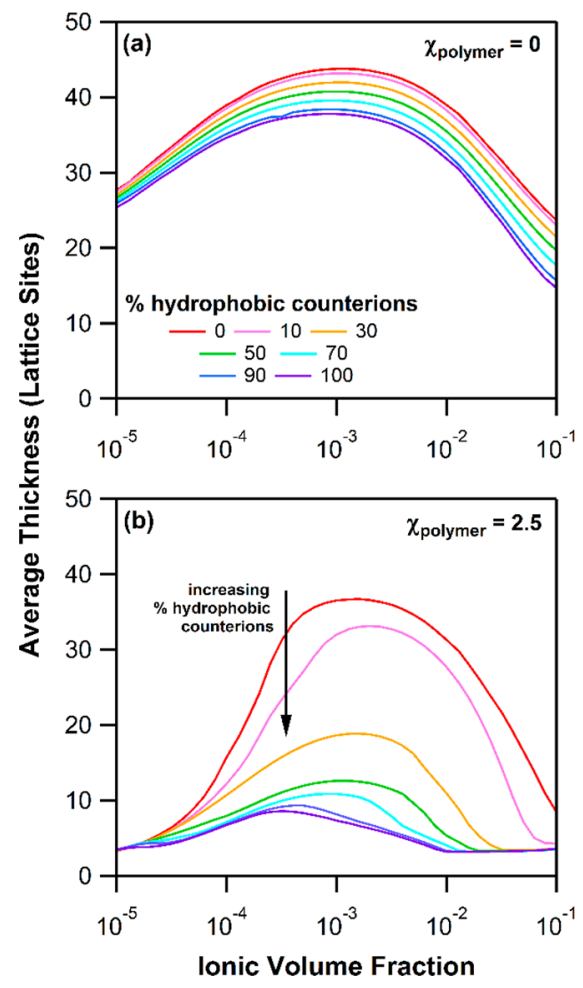

Figure 4. Average brush thickness as a function of ionic strength for hydrophilic $\left(\mathrm{a}, \chi_{\text {polymer }}=0\right)$ and hydrophobic $\left(\mathrm{b}, \chi_{\text {polymer }}=2.5\right)$ brushes at varying solvent compositions of hydrophilic $\left(\chi_{\text {counterion }}=0\right)$ and hydrophobic $\left(\chi_{\text {counterion }}=2.5\right)$ counterions as indicated, where $0 \%$ corresponds to only hydrophilic counterions and $100 \%$ is for only hydrophobic counterions.

hydrophobic polymer (see Figure 4b). Here, the thickness of the brush decreases monotonically with increasing presence of hydrophobic counterions, for a given bulk ionic volume fraction.

Figure 5 presents the volume fraction profiles for monomer segments as well as counterions for selected conditions for the most hydrophobic polymer. Figure S3 shows the profiles for a moderately hydrophobic brush $\left(\chi_{\text {polymer }}=1\right)$ and a hydrophilic brush $\left(\chi_{\text {polymer }}=0\right)$. From Figure 5, an understanding of the types of brush structures is gained. At 0 and $10 \%$ hydrophobic counterions, the brush is highly swollen, and the volume fraction profiles are characterized by long tails of polymer extending away from the substrate with a low volume fraction. From 0 to $50 \%$ hydrophobic counterions in solution, the brush transitions through an intermediate two-state phase in which a proportion of the chains are collapsed into a dense inner region at the substrate, while the remaining chains are highly extended away from it. With increasing ratios of hydrophobic counterions in the bulk solution, the brush thickness decreases as the swollen tail region of the brush completely disappears, and all the polymer chains reside in a dense slab at the substrate. This behavior is clearly shown by the inset plots shown within Figures $5 \mathrm{~b}$ and $5 \mathrm{~d}-\mathrm{g}$, in which the range for the volume fraction axis is much smaller. The volume fraction of counterions does not decrease to zero, but instead to its value in the bulk solution, which occurs after the polymer density reaches zero.

Close inspection of Figure 4 reveals that brush behavior in the presence of mixed salt solutions is more complex than the behavior in the presence of only single salt types. For example,
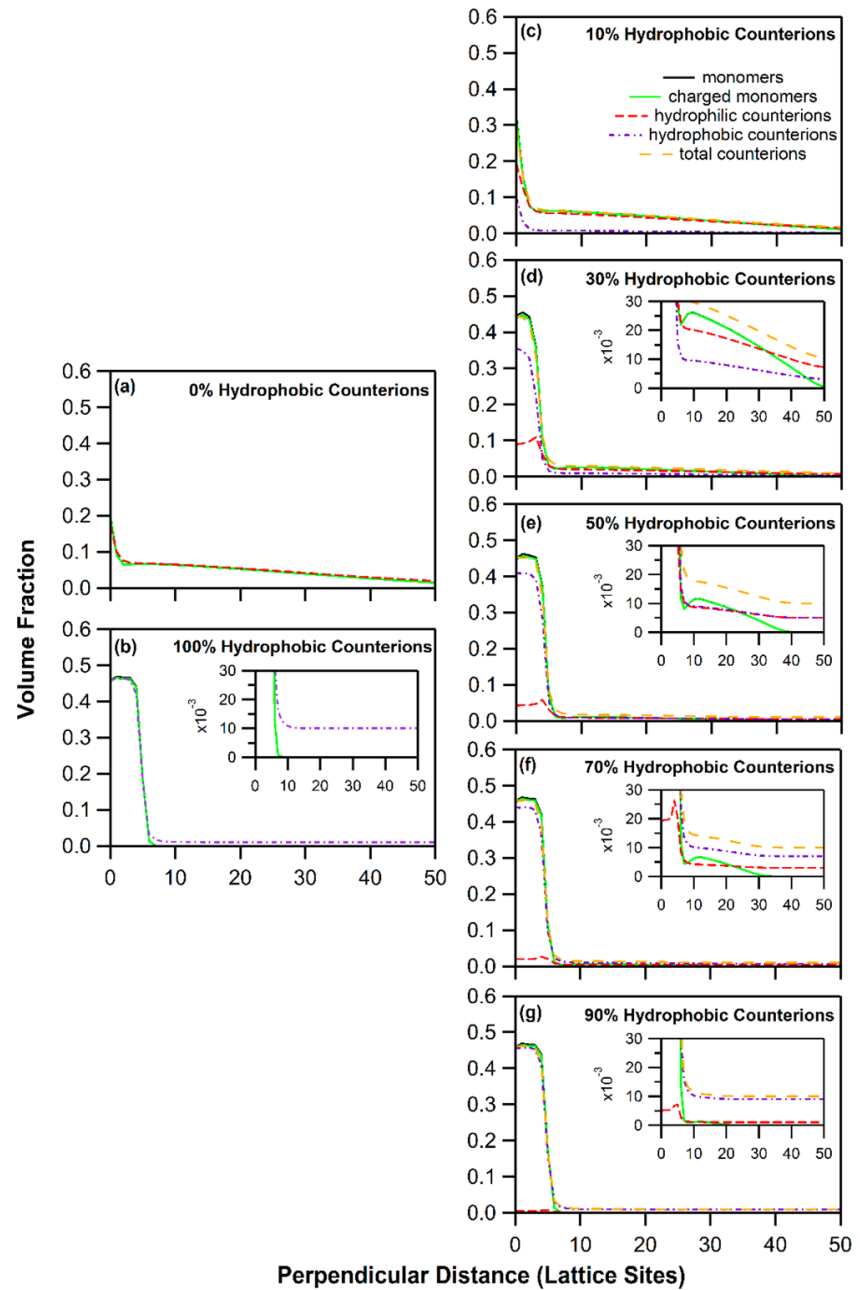

Perpendicular Distance (Lattice Sites)

Figure 5. Monomer and counterion volume fraction profiles for a hydrophobic weak polyelectrolyte brush $\left(\chi_{\text {polymer }}=2.5\right)$ immersed in mixed salt solutions composed of (a) $0 \%$, (b) $100 \%$, (c) 10\%, (d) $30 \%$, (e) $50 \%$, (f) $70 \%$, and $(\mathrm{g}) 90 \%$ hydrophobic $\left(\chi_{\text {counterion }}=2.5\right)$ counterions; the remaining percentage is made up of hydrophilic $\left(\chi_{\text {counterion }}=0\right)$ counterions. The bulk ionic volume fraction is $10^{-2}$. For $(b, d-g)$, inset plots are provided to show the behavior at small volume fractions.

comparison of Figure $2 \mathrm{~b}$ with Figure $4 \mathrm{~b}$ at the selected ionic volume fraction of $10^{-2}$ reveals that indeed the effect of mixed salts is much greater than one would expect on the basis of just the average behavior of the pure counterions (or the average Flory-Huggins interaction parameters of the counterions), which is explored in more detail below. This interesting behavior can be understood by considering the composition of counterions that are confined within the brush.

Figure 6 presents the percentage of hydrophobic counterions $\left(\chi_{\text {counterion }}=2.5\right)$ compared to hydrophilic $\left(\chi_{\text {counterion }}=0\right)$ counterions that are confined within the brush as a function of the percentage of hydrophobic to hydrophilic counterions present in the bulk solution for $\chi_{\text {polymer }}=0-2.5$. Figure $6 \mathrm{a}$ is data for a bulk ionic volume fraction of $10^{-2}$, while Figure $6 \mathrm{~b}$ is data for a bulk ionic volume fraction of $10^{-3}$, and comparison of the two reveals very little difference between the two data sets showing that bulk ionic strength has minimal influence on the composition of counterions confined within the brush (which is also true for other bulk ionic strengths). 


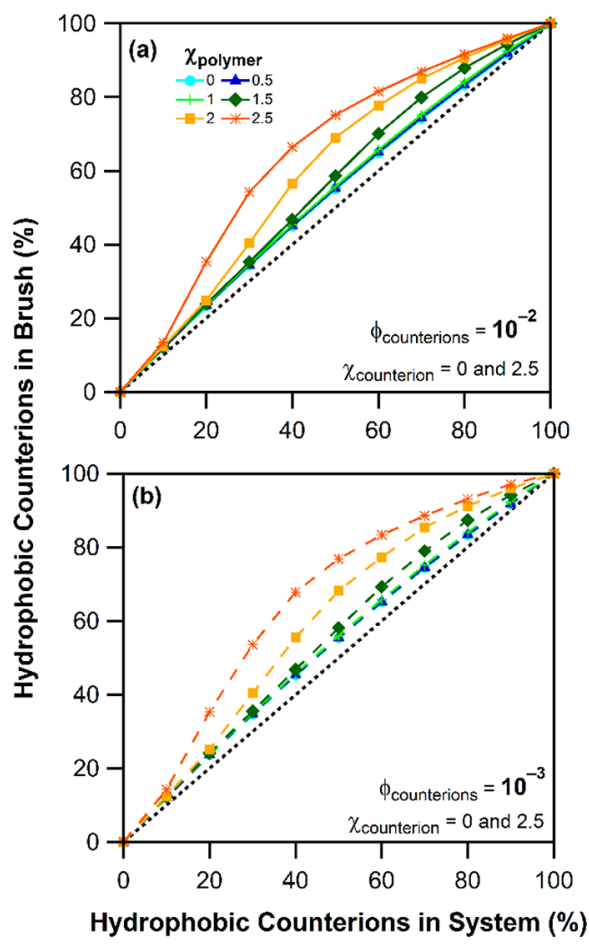

Figure 6. Percentage of hydrophobic counterions confined within the brush as a function of the percentage of hydrophobic anions in the bulk solvent for $\chi_{\text {polymer }}=0-2.5$. The bulk solvent is made up of hydrophilic $\left(\chi_{\text {counterion }}=0\right)$ and hydrophobic $\left(\chi_{\text {counterion }}=2.5\right)$ counterions; the composition ranges from 0 to $100 \%$. Results are given for bulk ionic volume fractions of (a) $10^{-2}$ and (b) $10^{-3}$. The black dotted line splits the axes symmetrically; the region above the line represents predominant confinement of the more hydrophobic counterions.

In Figure 6, the dotted line represents the case of ideal mixing or no specific interactions with the brush; e.g., at 50:50 hydrophobic:hydrophilic counterions in the bulk system, the composition of counterions within the brush is also 50:50. Therefore, deviations away from this line indicate more complex behavior. For the hydrophilic polymers, the confinement of hydrophobic counterions from the bulk solution is slightly favored over that of hydrophilic counterions. The hydrophobic counterions favor interaction with the brush as it allows them to interact less with the water molecules. However, the effect of this on average brush thickness is not strong as the polymer-solvent interaction is favored. As polymer hydrophobicity increases, the data diverge much further from the dotted line. In Figure 6a, if we take the example of when the bulk solution is composed of 50\% hydrophilic and 50\% hydrophobic counterions, we see that for $\chi_{\text {polymer }}=2.5$ the composition of counterions confined within the brush is $\sim 25 \%$ hydrophilic and $\sim 75 \%$ hydrophobic ions, respectively, at an ionic volume fraction of $10^{-2}$. Overall, the trend in the counterion composition within the brush is nonlinear. Figure S4 presents the case for $\chi_{\text {counterion }}=0$ and 1 and shows that the confinement of the moderately hydrophobic counterions (with interaction parameter of 1 ) is slightly favored over that of the hydrophilic counterions.

We now present a closer look at what impact the counterion composition within the brush has on the overall brush thickness response; here we will just consider the case of $\chi_{\text {counterion }}=0$ and 2.5. In Figure 7, the percentage change in brush thickness from its most swollen state (when in $100 \%$ hydrophilic ions) is

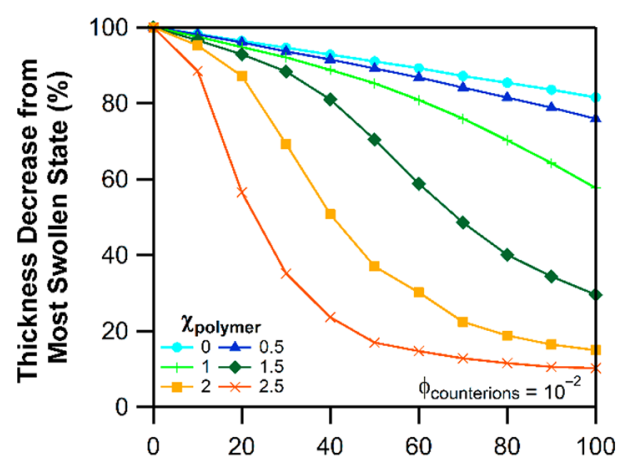

Hydrophobic Counterions in System (\%)

Figure 7. Percentage decrease in brush thickness from the most swollen state (i.e., in $100 \%$ hydrophilic ions, top left of graph) as a function of the percentage of hydrophobic counterions present in the bulk solution for $\chi_{\text {polymer }}=0-2.5$. In all cases, the bulk ionic volume fraction is $10^{-2}$.

plotted as a function of the percentage of hydrophobic counterions in the bulk solution for an ionic volume fraction of $10^{-2}$. The reduction in overall brush thickness upon the gradual introduction of hydrophobic counterions into the system (i.e., moving from top left toward the bottom right of the graph) is clear.

For the hydrophilic polymers, the decrease in brush thickness with increasing percentage of hydrophobic counterions in the bulk system is small. However, significant decreases in brush thickness are seen for the hydrophobic polymers $\left(\chi_{\text {polymer }}=2\right.$ and 2.5) and are greatest for $\chi_{\text {polymer }}$ of 2.5. For instance, if we consider the case of 50:50 hydrophobic:hydrophilic counterions, we see that for $\chi_{\text {polymer }}=0$ the average brush thickness is halfway between the most swollen and most collapsed states ( $0 \%$ and $100 \%$ of hydrophobic ions in solution, respectively). However, for the same case at $\chi_{\text {polymer }}=2.5$, the percentage decrease in brush thickness is much larger at $\sim 85 \%$, and the brush has almost reached the same thickness as in the case of $100 \%$ hydrophobic counterions. Indeed, substantial changes in brush swelling are even present with very small amounts of hydrophobic counterions in the system. Understanding this behavior requires investigating where within the brush the different counterions are confined.

Further analysis reveals the location of the different types of counterions confined within the brush. For this the brush is split into two regions: the inner region (close to the grafting surface, defined as volume fraction $>0.1$ ) and the tail region (polymer that extends into the bulk solvent, defined as volume fraction <0.1). Note that the choice of the volume fraction cutoff is arbitrary and does not affect the key conclusions drawn. Figure 8 presents the percentage of hydrophobic counterions that are confined within the inner region (a, volume fraction $>0.1)$ and the tail region $(b$, volume fraction $<0.1$ ) of the brush as a function of the percentage of hydrophobic counterions present in the bulk solution for the same conditions as in Figures 6a and 7. Figure S5 shows the percentage of total monomers with volume fraction $>0.1$ and therefore is a measure of brush conformation revealing that hydrophilic polymers $\left(\chi_{\text {polymer }}<0.5\right)$ are always extended with the vast majority of the monomers located in the tail region of the brush. At intermediate polymer hydrophobicities, most of the polymer still resides in the tail region of the brush, but for more hydrophobic polymers $\left(\chi_{\text {polymer }}>2\right)$ brush collapse is favored and most of the polymer is found within the inner 

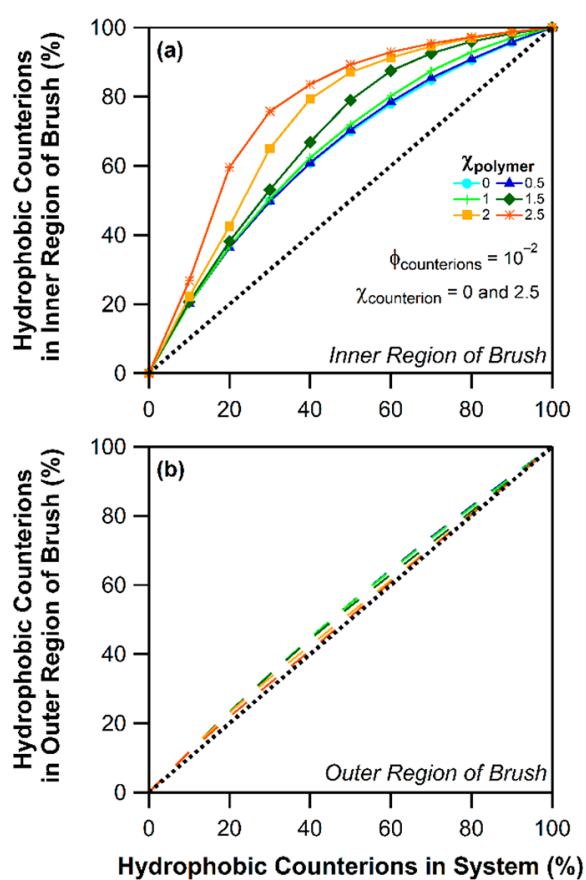

Figure 8. Percentage of hydrophobic counterions confined within (a) the inner region and (b) the tail region of the brush as a function of the percentage of hydrophobic counterions in the bulk solvent for $\chi_{\text {polymer }}=0-2.5$. In both plots, the black dotted line splits the axes symmetrically; the region above the line represents predominant confinement of the hydrophobic counterions.

region of the brush. The intriguing result is exposed by Figure $8 \mathrm{a}$ and demonstrations that hydrophobic counterions have a strong preference for the collapsed (inner) region of the brush. Conversely, in the tail region (Figure $8 \mathrm{~b}$ ), the composition of ions within the brush closely matches that of the bulk.

The entirety of these results can assist in designing experiments to look at the effect of mixed salts on polyelectrolyte brushes. One could envisage a carefully designed neutron reflectometry experiment that could elucidate brush profile while probing the counterions confined within the brush by using, for example, deuterated hydrophobic counterions. ${ }^{46}$ Simultaneous ellipsometry and QCM-D experiments would also be powerful as they are sensitive to the average and maximal extents of the brush, respectively. ${ }^{47}$

\section{CONCLUSIONS}

Numerical self-consistent field theory has been used to study the behavior of hydrophilic and hydrophobic weak polyelectrolyte brushes immersed in solutions of mixed electrolytes. A Flory-Huggins interaction parameter was assigned not just to the monomer segments but also to the counterions so that the strength of ion hydration could be varied, resembling the Hofmeister series. For mixed salts, the solvent contained two different counterion types, and the proportion of each was incrementally varied from $100 \%$ hydrophilic anions $\left(\chi_{\text {counterion }}\right.$ of 0 ) through to $100 \%$ hydrophobic anions $\left(\chi_{\text {counterion }}\right.$ of either 1 or 2.5$)$. Results showed that for hydrophobic polymers the confinement of the hydrophobic counterions was greatly favored over that of the hydrophilic counterions. The increased proportion of hydrophobic ions within the polyelectrolyte brush shifted it toward collapsed conformations, with the effect evident even at very small percentages of hydrophobic anions in solution. Moreover, the interior of the brush (closest to substrate) was where the hydrophobic ions were predominantly confined, while the composition of the tail region of the brush closely matched that the overlying solution. With these results we have shown that mixed salt solutions offer an additional mechanism to control the response of weakly charged polyelectrolytes.

\section{ASSOCIATED CONTENT}

\section{Supporting Information}

The Supporting Information is available free of charge on the ACS Publications website at DOI: 10.1021/acs.macromol.7b02244.

Definition of counterion confinement within the brush, supporting figures for the weak polybasic brush in the presence of hydrophilic $\left(\chi_{\text {counterion }}=0\right)$ and moderately hydrophobic $\left(\chi_{\text {counterion }}=1\right)$ counterions, including the polymer and counterion volume fraction profiles for $\chi_{\text {polymer }}=0$ and 1 , and last the percentage of monomers with volume fraction $>0.1$ (PDF)

\section{AUTHOR INFORMATION}

\section{Corresponding Author}

*(J.D.W.) E-mail j.d.willott@utwente.nl; phone +31 53 4893405 .

ORCID $\odot$

Joshua D. Willott: 0000-0003-1870-755X

Timothy J. Murdoch: 0000-0001-8727-0908

Frans A. M. Leermakers: 0000-0001-5895-2539

\section{Author Contributions}

" These authors contributed equally to the manuscript.

\section{Notes}

The authors declare no competing financial interest.

\section{ACKNOWLEDGMENTS}

W.d.V. and J.D.W. acknowledge finding support from the "Vernieuwingsimpuls" programme through project VIDI 723.015.003 (financed by The Netherlands Organization for Scientific Research, NWO). T.J.M. thanks AINSE Ltd. for providing financial assistance (Award - PGRA). The authors deeply thank Grant B. Webber for fruitful discussions.

\section{REFERENCES}

(1) Nordgren, N.; Rutland, M. W. Tunable Nanolubrication between Dual-Responsive Polyionic Grafts. Nano Lett. 2009, 9, 2984-2990.

(2) Fielding, L. A.; Edmondson, S.; Armes, S. P. Synthesis of pHResponsive Tertiary Amine Methacrylate Polymer Brushes and Their Response to Acidic Vapour. J. Mater. Chem. 2011, 21, 11773-11780.

(3) Willott, J. D.; Murdoch, T. J.; Humphreys, B. A.; Edmondson, S.; Webber, G. B.; Wanless, E. J. Critical Salt Effects in the Swelling Behavior of a Weak Polybasic Brush. Langmuir 2014, 30, 1827-1836.

(4) Willott, J. D.; Humphreys, B. A.; Murdoch, T. J.; Edmondson, S.; Webber, G. B.; Wanless, E. J. Hydrophobic Effects within the Dynamic $\mathrm{pH}$ Response of Polybasic Tertiary Amine Methacrylate Brushes. Phys. Chem. Chem. Phys. 2015, 17, 3880-3890.

(5) Willott, J. D.; Murdoch, T. J.; Humphreys, B. A.; Edmondson, S.; Wanless, E. J.; Webber, G. B. Anion-Specific Effects on the Behavior of pH-Sensitive Polybasic Brushes. Langmuir 2015, 31, 3707-3717.

(6) Chen, T.; Ferris, R.; Zhang, J.; Ducker, R.; Zauscher, S. StimulusResponsive Polymer Brushes on Surfaces: Transduction Mechanisms and Applications. Prog. Polym. Sci. 2010, 35, 94-112.

(7) Guenoun, P. Polyelectrolyte Brushes: Twenty Years After. In Functional Polymer Films; Wiley-VCH Verlag GmbH \& Co. KGaA: 2011. 
(8) Willott, J. D.; Murdoch, T. J.; Webber, G. B.; Wanless, E. J. Physicochemical Behaviour of Cationic Polyelectrolyte Brushes. Prog. Polym. Sci. 2017, 64, 52-75.

(9) Rodríguez-Ropero, F.; van der Vegt, N. F. A. Ionic Specific Effects on the Structure, Mechanics and Interfacial Softness of a Polyelectrolyte Brush. Faraday Discuss. 2013, 160, 297-309.

(10) Murdoch, T. J.; Willott, J. D.; de Vos, W. M.; Nelson, A.; Prescott, S. W.; Wanless, E. J.; Webber, G. B. Influence of Anion Hydrophilicity on the Conformation of a Hydrophobic Weak Polyelectrolyte Brush. Macromolecules 2016, 49, 9605-9617.

(11) Lyatskaya, Y. V.; Leermakers, F. A. M.; Fleer, G. J.; Zhulina, E. B.; Birshtein, T. M. Analytical Self-Consistent-Field Model of Weak Polyacid Brushes. Macromolecules 1995, 28, 3562-3569.

(12) Zhulina, E. B.; Borisov, O. V. Structure and Interaction of Weakly Charged Polyelectrolyte Brushes: Self-Consistent Field Theory. J. Chem. Phys. 1997, 107, 5952-5967.

(13) Zhulina, E. B.; Borisov, O. V. Poisson-Boltzmann Theory of pH-Sensitive (Annealing) Polyelectrolyte Brush. Langmuir 2011, 27, 10615-10633.

(14) Borisov, O. V.; Zhulina, E. B.; Birshtein, T. M. Diagram of the States of a Grafted Polyelectrolyte Layer. Macromolecules 1994, 27, 4795-4803.

(15) Israëls, R.; Leermakers, F. A. M.; Fleer, G. J. On the Theory of Grafted Weak Polyacids. Macromolecules 1994, 27, 3087-3093.

(16) Israëls, R.; Leermakers, F. A. M.; Fleer, G. J.; Zhulina, E. B. Charged Polymeric Brushes: Structure and Scaling Relations. Macromolecules 1994, 27, 3249-3261.

(17) Pryamitsyn, V. A.; Leermakers, F. A. M.; Fleer, G. J.; Zhulina, E. B. Theory of the Collapse of the Polyelectrolyte Brush. Macromolecules 1996, 29, 8260-8270.

(18) Witte, K. N.; Kim, S.; Won, Y. Y. Self-Consistent Field Theory Study of the Effect of Grafting Density on the Height of a Weak Polyelectrolyte Brush. J. Phys. Chem. B 2009, 113, 11076-11084.

(19) Nap, R.; Gong, P.; Szleifer, I. Weak Polyelectrolytes Tethered to Surfaces: Effect of Geometry, Acid-Base Equilibrium and Electrical Permittivity. J. Polym. Sci., Part B: Polym. Phys. 2006, 44, 2638-2662.

(20) Gong, P.; Wu, T.; Genzer, J.; Szleifer, I. Behavior of SurfaceAnchored Poly(acrylic acid) Brushes with Grafting Density Gradients on Solid Substrates: 2. Theory. Macromolecules 2007, 40, 8765-8773.

(21) Nap, R. J.; Tagliazucchi, M.; Szleifer, I. Born Energy, Acid-Base Equilibrium, Structure and Interactions of End-Grafted Weak Polyelectrolyte Layers. J. Chem. Phys. 2014, 140, 024910.

(22) Léonforte, F.; Welling, U.; Müller, M. Single-Chain-in-MeanField Simulations of Weak Polyelectrolyte Brushes. J. Chem. Phys. 2016, 145, 224902.

(23) Sudre, G.; Hourdet, D.; Creton, C.; Cousin, F.; Tran, Y. pHResponsive Swelling of Poly(acrylic acid) Brushes Synthesized by the Grafting Onto Route. Macromol. Chem. Phys. 2013, 214, 2882-2890.

(24) Mahalik, J. P.; Yang, Y.; Deodhar, C.; Ankner, J. F.; Lokitz, B. S.; Kilbey, S. M.; Sumpter, B. G.; Kumar, R. Monomer Volume Fraction Profiles in pH Responsive Planar Polyelectrolyte Brushes. J. Polym. Sci., Part B: Polym. Phys. 2016, 54, 956-964.

(25) Swann, J. M. G.; Bras, W.; Topham, P. D.; Howse, J. R.; Ryan, A. Effect of the Hofmeister Anions upon the Swelling of a SelfAssembled pH-Responsive Hydrogel. Langmuir 2010, 26, 1019110197.

(26) Willott, J. D.; Murdoch, T. J.; Webber, G. B.; Wanless, E. J. Nature of the Specific Anion Response of a Hydrophobic Weak Polyelectrolyte Brush Revealed by AFM Force Measurements. Macromolecules 2016, 49, 2327-2338.

(27) Murat, M.; Grest, G. S. Structure of a Grafted Polymer Brush: a Molecular Dynamics Simulation. Macromolecules 1989, 22, 40544059 .

(28) Scheutjens, J. M. H. M.; Fleer, G. J. Statistical Theory of the Adsorption of Interacting Chain Molecules. 1. Partition Function, Segment Density Distribution, and Adsorption Isotherms. J. Phys. Chem. 1979, 83, 1619-1635.

(29) Wijmans, C. M.; Scheutjens, J. M. H. M.; Zhulina, E. B. SelfConsistent Field Theories for Polymer Brushes: Lattice Calculations and an Asymptotic Analytical Description. Macromolecules 1992, 25, 2657-2665.

(30) Zhulina, E. B.; Leermakers, F. A. M. A Self-Consistent Field Analysis of the Neurofilament Brush with Amino-Acid Resolution. Biophys. J. 2007, 93, 1421-1430.

(31) de Vos, W. M.; Leermakers, F. A. M.; Lindhoud, S.; Prescott, S. W. Modeling the Structure and Antifouling Properties of a Polymer Brush of Grafted Comb-Polymers. Macromolecules 2011, 44, 23342342.

(32) Edwards, S. F. The Statistical Mechanics of Polymers with Excluded Volume. Proc. Phys. Soc., London 1965, 85, 613.

(33) Halperin, A.; Kröger, M. Collapse of Thermoresponsive Brushes and the Tuning of Protein Adsorption. Macromolecules 2011, 44, 6986-7005.

(34) Lyklema, J. Fundamentals of Interface and Colloid Science; Academic Press: London, 1991.

(35) Emileh, A.; Vasheghani-Farahani, E.; Imani, M. Swelling Behavior, Mechanical Properties and Network Parameters of $\mathrm{pH}-$ and Temperature-Sensitive Hydrogels of $\operatorname{Poly}((2$-dimethyl amino) Ethyl Methacrylate-co-Butyl Methacrylate. Eur. Polym. J. 2007, 43, 1986-1995.

(36) Salis, A.; Ninham, B. W. Models and Mechanisms of Hofmeister Effects in Electrolyte Solutions, and Colloid and Protein Systems Revisited. Chem. Soc. Rev. 2014, 43, 7358-7377.

(37) Biesalski, M.; Johannsmann, D.; Ruhe, J. Electrolyte-Induced Collapse of a Polyelectrolyte Brush. J. Chem. Phys. 2004, 120, 88078814.

(38) Bibi, I.; Siddiq, M. Conformational Transition of Poly(vinylbenzyltrimethylammonium chloride) (PVBTMAC) Brush in the Presence of Hofmeister Anions. J. Polym. Res. 2012, 19, 1-5.

(39) Attili, S.; Borisov, O. V.; Richter, R. P. Films of End-Grafted Hyaluronan are a Prototype of a Brush of a Strongly Charged, Semiflexible Polyelectrolyte with Intrinsic Excluded Volume. Biomacromolecules 2012, 13, 1466-1477.

(40) Pryamitsyn, V.; Leermakers, F.; Fleer, G.; Zhulina, E. Theory of the Collapse of the Polyelectrolyte Brush. Macromolecules 1996, 29, 8260-8270.

(41) Carrillo, J.-M. Y.; Dobrynin, A. V. Morphologies of Planar Polyelectrolyte Brushes in a Poor Solvent: Molecular Dynamics Simulations and Scaling Analysis. Langmuir 2009, 25, 13158-13168.

(42) Tagliazucchi, M.; de la Cruz, M. O.; Szleifer, I. Self-Organization of Grafted Polyelectrolyte Layers via the Coupling of Chemical Equilibrium and Physical Interactions. Proc. Natl. Acad. Sci. U. S. A. 2010, 107, 5300-5305.

(43) He, G.-L.; Merlitz, H.; Sommer, J.-U. Molecular Dynamics Simulations of Polyelectrolyte Brushes under Poor Solvent Conditions: Origins of Bundle Formation. J. Chem. Phys. 2014, 140, 104911.

(44) Günther, J. U.; Ahrens, H.; Förster, S.; Helm, C. A. Bundle Formation in Polyelectrolyte Brushes. Phys. Rev. Lett. 2008, 101, 258303.

(45) Hur, J.; Witte, K. N.; Sun, W.; Won, Y.-Y. On the Origins of the Salt-Concentration-Dependent Instability and Lateral Nanoscale Heterogeneities of Weak Polyelectrolyte Brushes: Gradient Brush Experiment and Flory-Type Theoretical Analysis. Langmuir 2010, 26, 2021-2034.

(46) Prabhu, V. M.; Vogt, B. D.; Wu, W.-1.; Douglas, J. F.; Lin, E. K.; Satija, S. K.; Goldfarb, D. L.; Ito, H. Direct Measurement of the Counterion Distribution within Swollen Polyelectrolyte Films. Langmuir 2005, 21, 6647-6651.

(47) Zhuang, P.; Dirani, A.; Glinel, K.; Jonas, A. M. Temperature Dependence of the Surface and Volume Hydrophilicity of Hydrophilic Polymer Brushes. Langmuir 2016, 32, 3433-3444. 